\title{
Association testing of Kuwaiti subset admixture population using mass and targeted SNP genotyping technologies
}

\section{Introduction}

With the increasing density of SNP markers, attention is rapidly shifting from traditional linkage and positional cloning methods for gene discovery to association methods for complex diseases ${ }^{1,2}$. Because complex traits presumably arise from multiple interacting genes located throughout the genome, it would be appropriate to search for sets of marker loci in different genes and to analyze these markers jointly rather than testing each marker in isolation ${ }^{3}$. Thus, identification of the causal mutation(s) can be difficult, as there are potentially a large number of polymorphic sites within a gene, many of which may appear to be associated with a disease simply because they are in linkage disequilibrium with a causal mutation but have no functional significance on their own.

Interest in SNPS and SNP-based association analyses are not likely to diminish soon. However, if progress in SNP-based initiatives is to be made, it is important to recognize and document the potential strengths and weaknesses of analysis methods making use of SNP applications ${ }^{2,4}$.

\section{Objectives}

To determine association of functional obesity-alleles with metabolic profiles in a Kuwaiti admixture subset using two SNP genotyping technologies, mass genotyping and targeted genotyping.

\section{Methods}

Due to polygenic nature of obesity, we followed the multiple markers testing approach by choosing 29 SNPs from 8 genes to increase the power of gene mapping detected. SNPs were selected manually based on literature, then shortlisted with computational tool. A total of 273 samples were genotyped using both methods, mass genotyping using Omni Express Bead chips and targeted genotyping using real-time PCR (Figure 1). SNP Data were analyzed using SNAP, PLINK and Golden Helix to determine OR, correlation trend test, Simple Logistic Regression, Cochran- Armitage trend test and Multifactor Dimensionality Reduction.

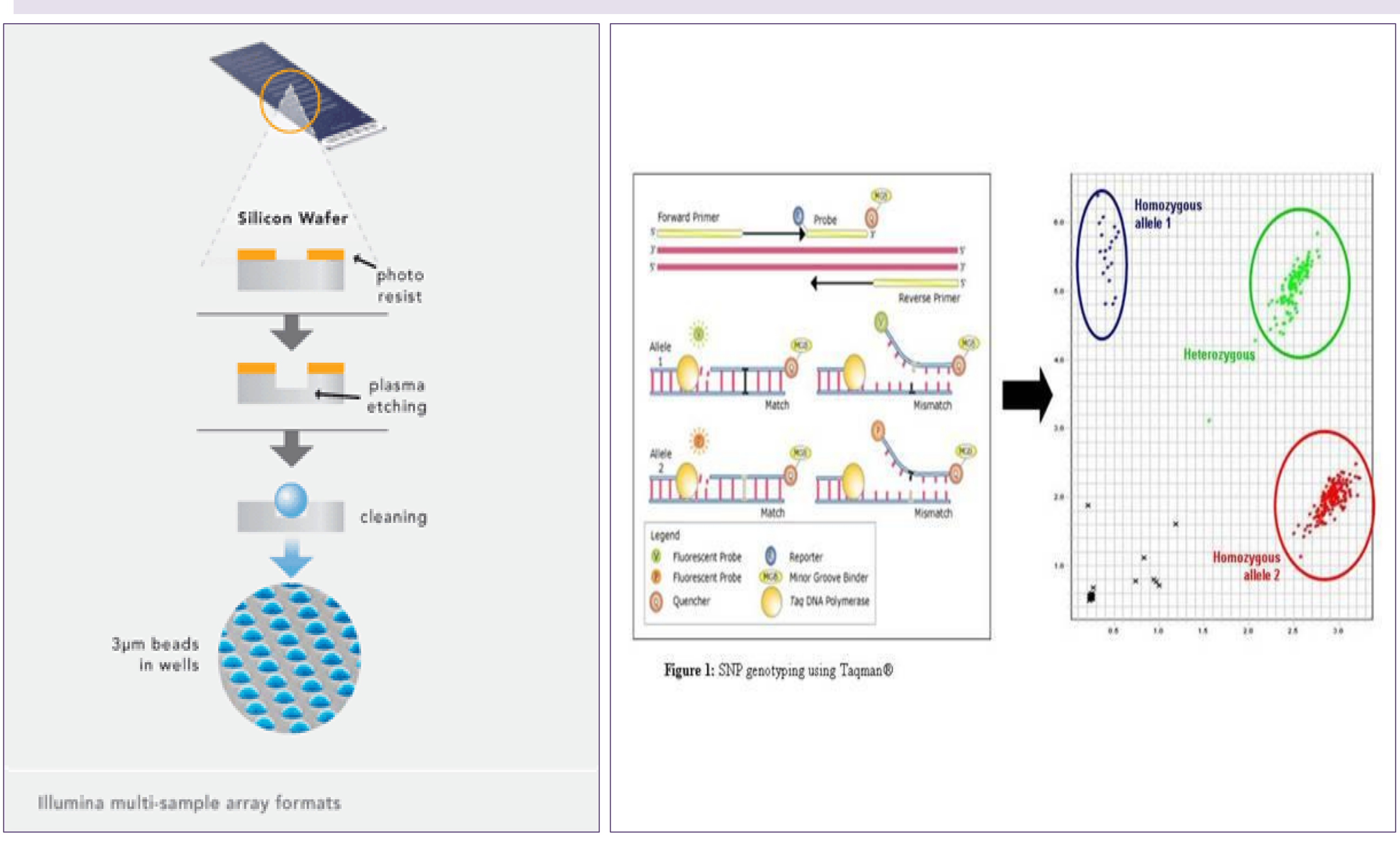

Figure1. Mass Genotyping using Omni Express Bead Chips vs. Targeted Genotyping using Real-time PCR technology.

\section{Results}

A total of 25/29 genotype counts of obesity-related SNPs were imprinted in the bead array chip while, only 4 genotype counts of SNPs were not included. Internal SNP quality checking was done for rs2076213, rs1435703, rs2294918, rs1421085, rs5219 and rs1801282 directly with Bead Array data. SNP quality check for rs2274459, rs9941349, rs6726292, rs1558902 were found out using SNAP tool by taking their high LD partners.

\section{Results}

We observed that rs2274459, rs1435703 and rs2076212 are associated with obesity at a P-value $0.045,0.034$ and 0.031 , respectively when $\mathrm{BMI}$ is taken as binary trait (Table 1). As for rs2274459, it was not in HWE and effected by excess rare homozygous in our population and was inconsistent with the Bead Array data; therefore, we consider only the two SNPs rs1435703 and rs2076212 as potential markers for obesity in Kuwaiti admixture population (as shown in from the P-value distribution in Figure 2).

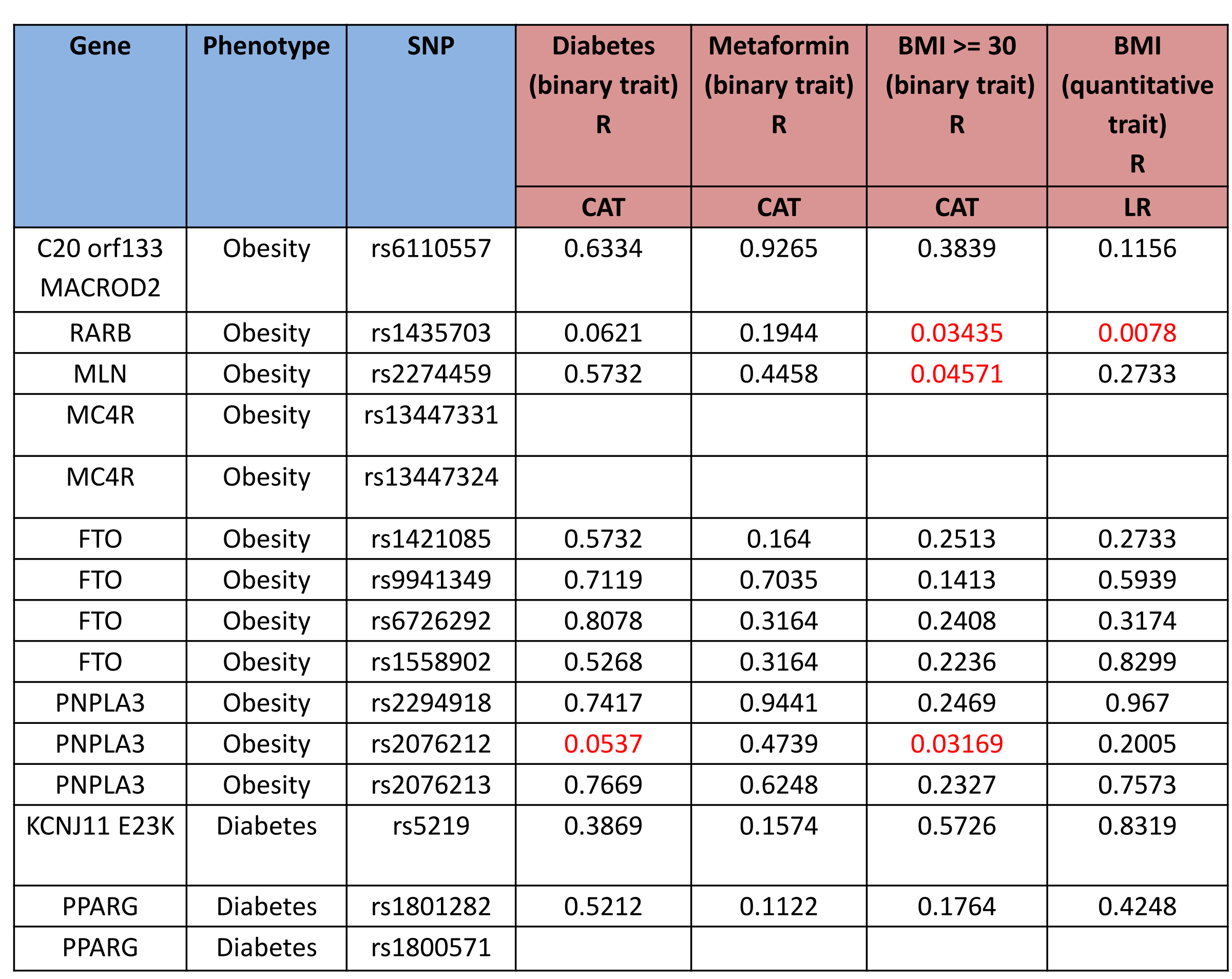
Table 1: Statistical diabetes, BMI and metformin status taken as binary status in Kuwaiti population. CAT: CochranArmitage trend test, $L R$ : Linear regression, $R: R$ script

$P$-value distribution of SNPS with statistical significance test

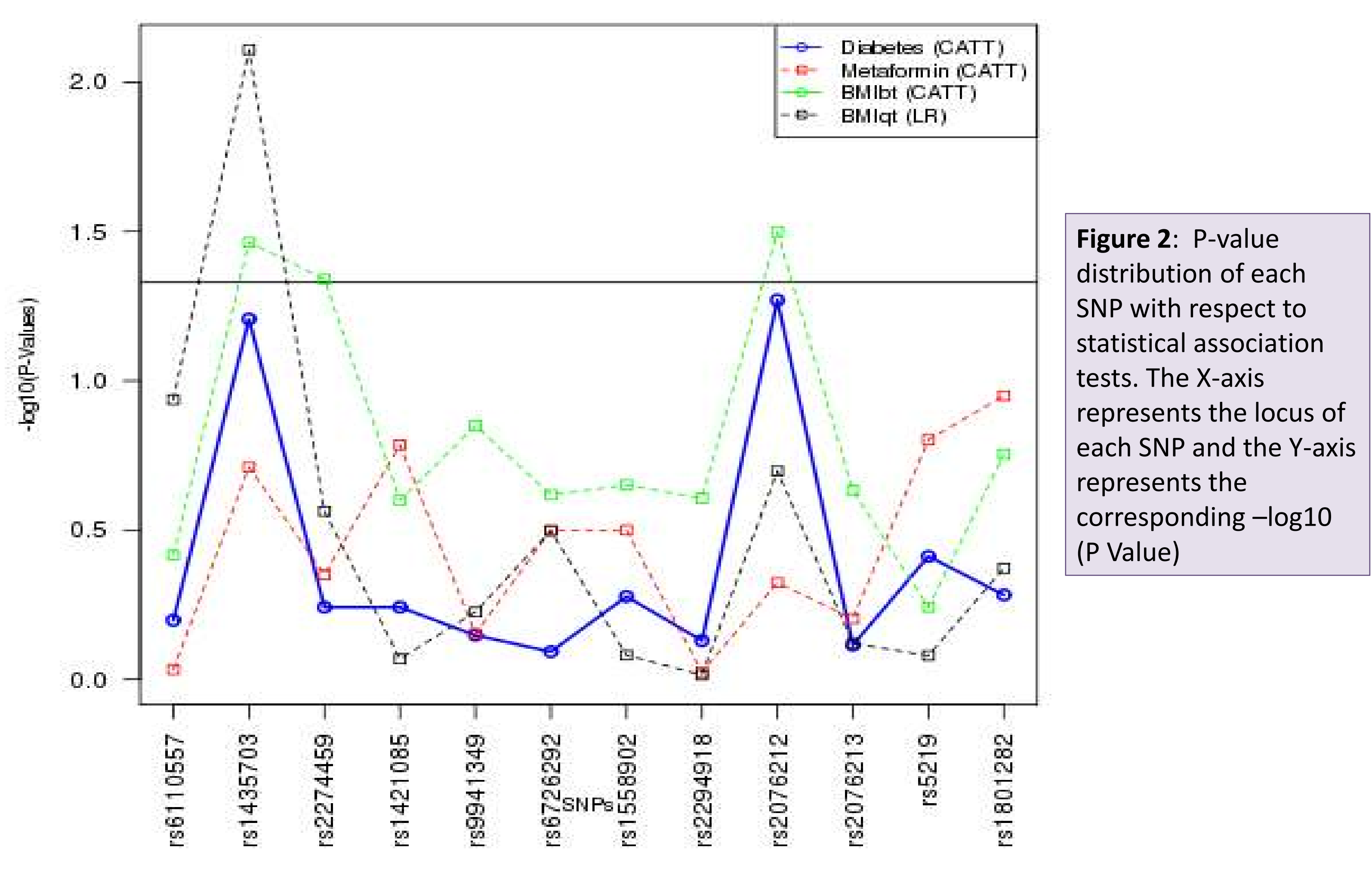

Conclusions

$>$ Both methods had their limitations, mass genotyping technology uses certain ethnicities for marker selection, while, targeted genotyping takes more time and effort. However, our data analysis from both methods showed that combinatorial effect of rs 13447324 with interaction system of rs14211085, rs1558902, rs2294918 provide greater risk assessment of obesity in Kuwaiti subset admixture population sample.

$>$ We consider only the two SNPs rs1435703 and rs2076212 as potential markers for obesity in Kuwaiti admixture population

\section{Acknowledgement}

This study was funded by Dasman Diabetes Institute, project number RA-2010-005

\section{References}

1. Risch $\mathrm{N}$ and Merikangas $\mathrm{K}$. The future of genetic studies of complex human diseases. Science 1996; 273: 1516-1517.

2. Schork NJ, Nath SK, Fallin D et al. Linkage disequilibrium analysis of biallelic DNA markers, human qunatitative trait loci, and threshold-defined case and control subjects. Am J Hum Genet 2000; 67: 1208-1218.

3. Fallin $D$, Cohen $A$, Essioux $L$ et al. Genetic analysis of case/control data using estimated haplotype frequencies: Application of APOE locus variation and Alzheimer's disease. Genome Res 2001; 11: 143-151.

4. Chakravarti A. It's raining SNPs, hallelujah? Nat Genet 1998; 19: 216-217. 